\title{
DAPPER: A Low Power, Dual Amperometric and Potentiometric Single-Channel Front End
}

\author{
Daryl $\mathrm{Ma}^{\star}$, Sara S. Ghoreishizadeh ${ }^{\dagger}$, Pantelis Georgiou ${ }^{\star}$ \\ ${ }^{\star}$ Dept. of Electrical \& Electronic Eng., Centre for Bio-Inspired Technology, Imperial College London, UK \\ ${ }^{\dagger}$ Aspire Centre for Rehabilitation and Assitive Technology, University College London, UK \\ Email: \{darylma, pantelis\}@imperial.ac.uk, s.ghoreishizadeh@ucl.ac.uk
}

\begin{abstract}
DAPPER is a front end system capable of simultaneous amperometric and potentiometric sensing proposed for lowpower multi-parameter analysis of bio-fluids such as saliva. The system consists of two oscillator circuits, generating a frequency relative to their sensed current and voltage signals. These signals are then mixed together to produce a single channel output that can be transmitted through backscattering (load-shift keying). The entire system consumes $40 \mu \mathrm{W}$ from a $1.4 \mathrm{~V}$ supply. The linear ranges of potentiometry and amperometry circuits are $0.4 \mathrm{~V}-1 \mathrm{~V}$ and $250 \mathrm{pA}-5.6 \mu \mathrm{A}(87 \mathrm{~dB})$, and their input referred noise is $1.7 \mu \mathrm{V}$ and 44.6fA, respectively.
\end{abstract}

\section{INTRODUCTION}

Interest in applications for wireless electrochemical sensing has grown over the past decade [1]. Its popularity stems from its low cost, as well as its ability to be made sensitive and selective to analytes to provide a real-time analysis of biofluids. In addition to the proliferation of smartphones, the evolution of Internet of Things (IoT) allows the possibility of introducing wireless electrochemical sensors into everyday life. These biosensors allow for a point-of-care approach to diagnosing patients, thus allowing for timely intervention.

The two most common transduction mechanisms for electrochemical sensing are potentiometry and amperometry. Through the use of ion-sensitive electrodes coupled with a potentiometric readout, the detection of ions such as potassium, sodium and $\mathrm{pH}$ is made possible. Additionally, CMOSbased ISFET arrays have been demonstrated for use with potentiometric sensing [2]. Amperometry is typically used for the detection of small biomolecules (lactate, ATP, glucose and cholesterol). There are numerous commercially available electrochemical glucose sensors, for instance the Enlite ${ }^{\mathrm{TM}}$ from Medtronic [3].

The most common biofluids currently used for medical diagnostics and monitoring in laboratories and at home are blood and interstitial fluids. These are invasive and expensive to collect. Saliva is a non-invasive alternative to blood in reflecting the state of health [4]. It is known that levels of most salivary biomolecules correlate with serum [5]. Saliva analysis provides vast potential for research in particular by facilitating continuous measurement of biomarkers. This can be achieved through a miniature intraoral wearable system equipped with an electrochemical sensor that allows realtime measurement of biomolecules over a sustained time. Challenges include detection from the whole biofluid that may contain potentially interfering molecules and the variability of

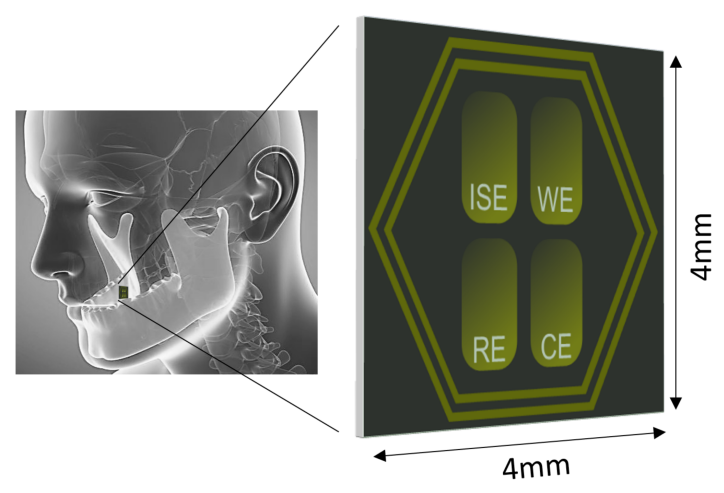

Fig. 1: Envisaged placement of the wireless sensor

the environment, such as variable temperature and $\mathrm{pH}$ of saliva that affects the performance of the electrochemical sensor [6]. The salivary $\mathrm{pH}$ has a circadian rhythm which is additionally affected by the patients diet, oral health condition, diabetes, gastric acid reflux, etc [7]. Therefore, readings acquired by electrochemical biomolecule sensors need to be calibrated with the $\mathrm{pH}$. Concurrent amperometric and potentiometric measurement is necessary for monitoring different types of analytes for co-analysis and to allow sensor calibration [8].

Few systems have been introduced to obtain both amperometry and potentiometry readings. For example, Sun et al [9] and Jiang et al [10] describe reconfigurable circuits that can be switched into potentiometric or amperometric modes at different moments in time. However, simultaneous potentiometric and amperometric readings, which is essential to capture instantaneous changes in $\mathrm{pH}$ for accurate calibration and reliable analysis [11], have yet to be achieved in literature.

This paper presents a Dual Amperometric and Potentiometric Power Efficient instrumentation (DAPPER). This system is an analog front end circuit that performs concurrent potentiometry and amperometry sensing, producing a single output by combining the two readings. The circuit consumes low power to allow wireless powering, for example using the circuit reported in [12]. Future work would involve transmitting the output data of this circuit wirelessly through backscattering (load shift keying).

The next section describes the design of the system, along with the rationale behind the choices made for the topologies. Section III demonstrates the simulation results for each block followed by the conclusion and future work. 


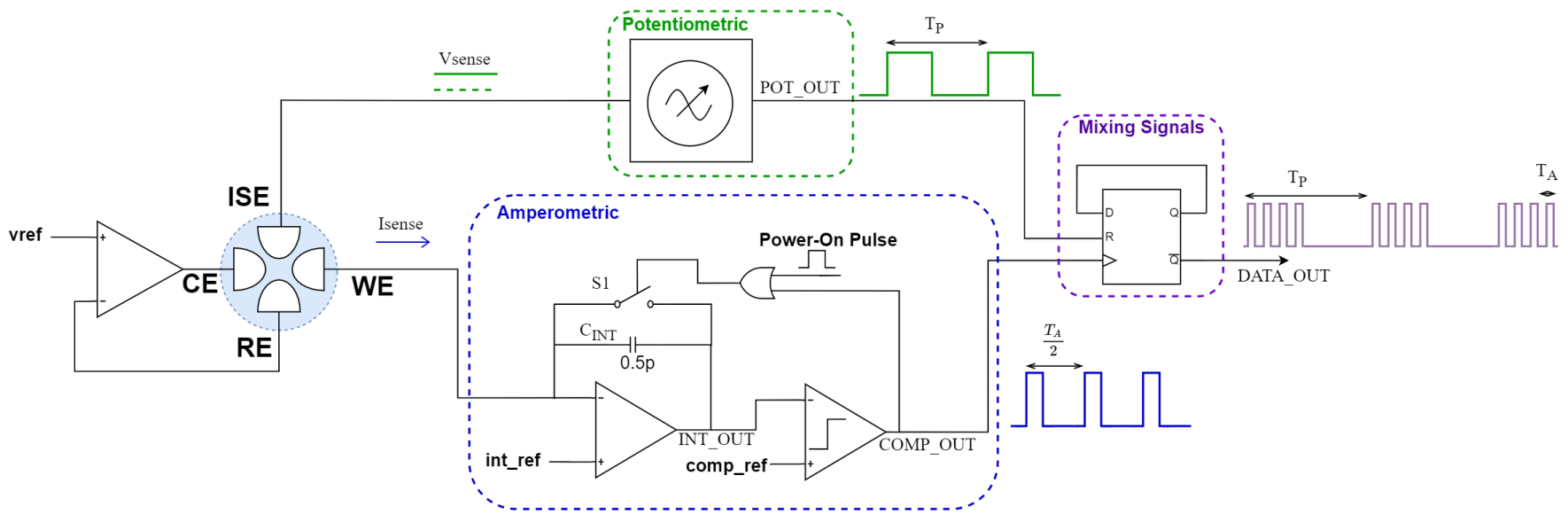

Fig. 2: The block diagram of DAPPER, the proposed dual amperometric and potentiometric front end

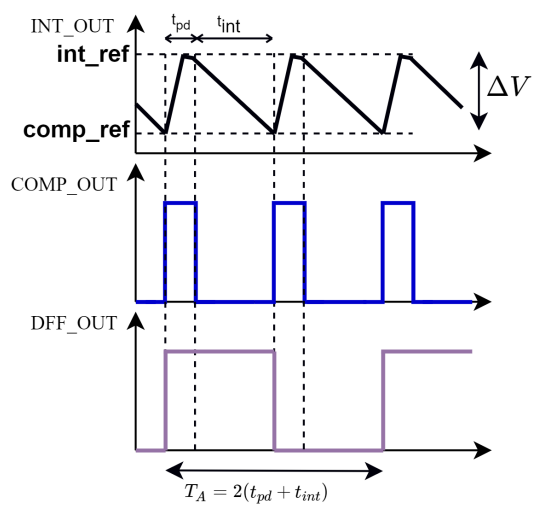

Fig. 3: Timing Diagram of Amperometric Circuit

\section{SySTEM Design}

DAPPER is illustrated in Fig. 2. The envisaged electrochemical cell consists of four electrodes: working electrode (WE) for amperometric sensing, an ion-selective electrode (ISE) for potentiometric measurement, a shared reference electrode (RE) and a counter electrode (CE). This electrode configuration takes inspiration from [10].

An amplifier in negative feedback drives the counter electrode (CE) and shared reference electrode (RE). Both the amperometric and potentiometric circuits transduce their inputs into output frequencies. The outputs of the two circuits are then mixed through the use of a D flip-flop to be transmitted outside on a single channel. The reference voltages $\mathbf{v}_{-}$ref, int_ref and comp_ref are provided externally, along with a power-on pulse to start the circuit. The circuit has four main blocks which will be detailed in this section.

\section{A. Amperometric Circuit}

The amperometric circuit consists of a switch-capacitor integrator and a comparator in a feedback loop. The circuit generates a square waveform with a frequency proportional to the input current. The integrator accumulates the input current on a capacitor when S1 is open. The reset clock of the capacitor is provided by the comparator to ensure the output voltage of the integrator is within a specific voltage range.
The amperometric circuit has two phases: integration and reset. The power-on-reset pulse sets the circuit in integration mode at system start-up. During the reset phase, COMP_OUT is high and S1 is closed. The integrator is configured as a voltage buffer, and INT_OUT rises to the input reference int_ref. During the integration phase where COMP OUT is low, $\mathrm{S} 1$ is open, the capacitor discharges and INT_O OUT falls to the comparator reference comp_ref. Once it reaches comp_ref, COMP OUT goes high, and closes S1 after a propagation delay from the output of the comparator to S1. This then starts the reset phase all over. The waveforms that occur throughout the circuit are presented in Fig. 3.

The comparator output is fed into the clock input of the $\mathrm{D}$ flip-flop. This is utilised as a divide-by-2 counter to obtain a $50 \%$ duty cycle, making it easier to demodulate at the backend.

Each period of the output pulse width consists of $t_{p d}$, the propagation delay from the time the comparator input crosses the threshold voltage to when S1 is closed, and $t_{\text {int }}$, the integration time. The output period of the DFF can be calculated as:

$$
T_{D F F_{-} \text {OUT }}=2\left(t_{p d}+t_{i n t}\right)
$$

From the equation for the charge in a switched-capacitor integrator, we obtain the integration time in Eq. 2, where $C_{I N T}$ is the feedback capacitor value, $\Delta \mathrm{V}$ is the difference in voltage between int_ref and comp_ref, and $I_{i n}$ is the input current:

$$
t_{i n t}=\frac{C_{I N T} \Delta V}{I_{i n}}
$$

Putting the equations together, we obtain an expression for the output frequency at the D flip-flop, $f_{o u t, a m p}$, as a function of input current in Eq. 3.

$$
f_{\text {out }, a m p}=\frac{I_{\text {in }}}{2\left(t_{p d} I_{\text {in }}+C_{I N T} \Delta V\right)}
$$

This shows the output frequency varies in a non-linear fashion due to the non-zero propagation delay $t_{p d}$ of the circuit. However, this could be estimated to a linear equation 
when $t_{p d}$ is small compared with $t_{i n t}$.

\section{B. Potentiometric Circuit}

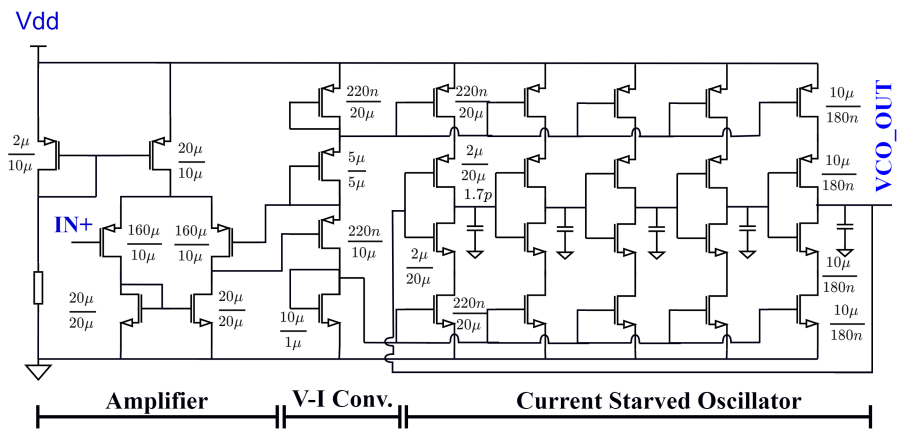

Fig. 4: Potentiometric Frontend Consisting of Current Starved Oscillator and Differential Pair

The potentiometric circuit serves to convert the input voltage read between RE and ISE into a frequency. Fig. 4 shows the potentiometric circuit where IN+ connects to ISE. This voltage input is passed through a differential input amplifier, before a central buffer is used to convert this voltage into a current. PMOS and NMOS current mirrors are used to bias the current sink and source transistors for the current starved ring oscillator. Extra capacitors are added after each stage to further reduce the frequency of the oscillators.

\section{Mixing the signals}

The output signals of the potentiometry and amperometry circuits are mixed using a D flip-flop with an asynchronous active-low reset. Fig. 5 demonstrates the mixing of the different waveforms. The figure describes a possible situation that arises when the potentiometric output is high during a low edge for the amperometric output. $t_{a}$ is the time when the potentiometric signal goes high, while $t_{e}$ is the time when the mixed signal goes high. $t_{b}$ captures the point where the potentiometric output goes low.

By detecting the edges in the mixed signal and calculating the edge-to-edge time difference, it is possible to reconstruct the amperometric data for $T_{A}$. To ensure at least two rising edges of $T_{A}$ are detected on the mixed signal, $T_{P}$ has to

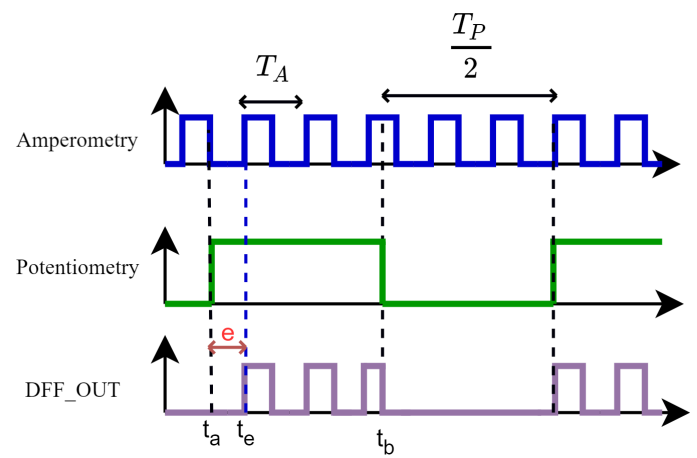

Fig. 5: Mixing Timing Diagram with the amperometric output, potentiometric output, and the mixed signals obtained, along with the error $t_{e}-t_{a}$

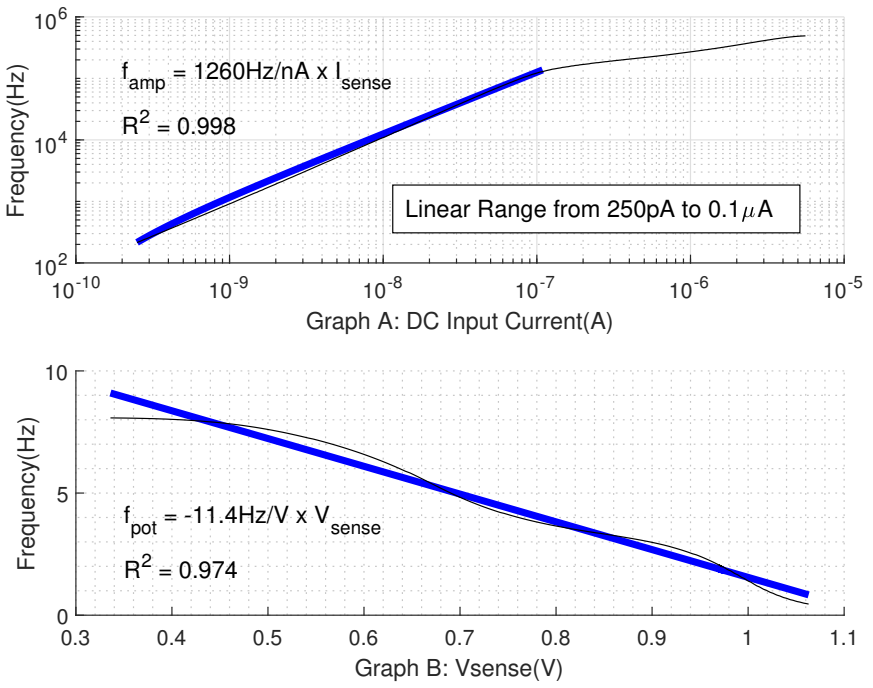

Fig. 6: A: Output frequency of amperometric circuit at D flipflop when potentiometry is deactivated for DC input current. B: Output frequency of potentiometric circuit for DC input voltage.

be chosen to be at least 4 times higher than $T_{A}$. For the potentiometric signal which occurs at a lower frequency, there exists an error arising from the phase difference. The received period of $T_{P}$ is:

$$
\frac{T_{P, \text { received }}}{2}=t_{b}-t_{e}=\frac{T_{P}}{2}-e
$$

where $t_{e}-t_{a}<T_{A}$. This becomes $\frac{t_{e}-t_{a}}{T_{P}}<\frac{T_{A}}{T_{P}}$, which allows us to deduce the percentage error. Thus, we have the requirement that the amperometric frequency is at least an order of magnitude $(\times 10)$ larger than the potentiometric frequency, which gives a maximum error for $T_{P, \text { received }}$ to be $10 \%$.

In addition, the input clock voltage threshold of the D flip-flop and the comparator are designed to only trigger for currents above the $250 \mathrm{pA}$ level. This is done by carefully designing the dimensions of the input transistors of the D flipflop. Hence, lower current values will not be detected.

\section{Amplifier}

The topology of the amplifier in Fig. 4 is used for both amperometric circuits and potentiometric circuits, and is a single stage differential input amplifier. PMOS transistors with long lengths are used as input transistors minimise the noise. The open-loop gain of the amplifier is $54 \mathrm{~dB}$ with a phase margin of $77^{\circ}$ (with no load connected). The maximum power consumption, power supply rejection ratio, common-mode rejection ratio and the $-3 \mathrm{~dB}$ bandwidth from simulations for the amplifier are $6 \mu \mathrm{W}, 52 \mathrm{~dB}, 63 \mathrm{~dB}$ and $10 \mathrm{kHz}$ respectively. The integrated input-referred voltage noise over the $-3 \mathrm{~dB}$ bandwidth of the amplifier $1.7 \mu \mathrm{V}$. 
TABLE I: Comparison with state-of-the-art

\begin{tabular}{|c|c|c|c|c|c|c|c|c|}
\hline Paper & $\begin{array}{l}\text { Technology } \\
\text { (nm) }\end{array}$ & $\begin{array}{l}\text { Supply } \\
\text { (V) }\end{array}$ & $\begin{array}{c}\text { Power } \\
(\mu \mathbf{W})\end{array}$ & $\begin{array}{c}\text { Input Referred } \\
\text { Noise }\end{array}$ & $\begin{array}{c}\text { Dynamic } \\
\text { Range } \\
\text { (dB) }\end{array}$ & $\begin{array}{c}\text { Size } \\
\left(\mathbf{m m}^{2}\right)\end{array}$ & $\begin{array}{l}\text { Trans. } \\
\text { Mech. }\end{array}$ & $\begin{array}{l}\text { Conc. } \\
\text { Trans. }\end{array}$ \\
\hline [13] & 350 & 3.3 & 9300 & $0.47 \mathrm{pA}$ & 156 (amp.) & 10.08 & Amp. & No \\
\hline [15] & NA & 3.3 & 3600 & $21.6 \mathrm{mV}$ & NA & 140 & Pot. & No \\
\hline [10] & 65 & 0.9 & 0.97 & $\begin{array}{l}0.5 \mathrm{mV} \text { (pot.) } \\
2.5 \mathrm{nA} \mathrm{(amp.)}\end{array}$ & $\begin{array}{c}43 \text { (pot.) } \\
30.1 \text { (amp.) }\end{array}$ & 1.275 & Amp.\&Pot. & No \\
\hline [17] & 350 & 1.4 & 92 & $1.8 \mu \mathrm{V}$ & 66 (pot.) & 2.1 & Pot. & No \\
\hline [18] & NA & $2.8-3.6$ & 990 & $\begin{array}{c}1.6 \mu \mathrm{V} \text { (pot.) } \\
0.05 \mathrm{nA} \text { (amp.) }\end{array}$ & $\begin{array}{c}121 \text { (pot.) } \\
155 \text { (amp.) }\end{array}$ & 15.12 & Amp.\& Pot. & No \\
\hline This Work & 180 & 1.4 & 40 & $\begin{array}{c}1.7 \mu \mathrm{V} \text { (pot.) } \\
44.6 \mathrm{fA} \text { (amp.) }\end{array}$ & $\begin{array}{l}62 \text { (pot.) } \\
87 \text { (amp.) }\end{array}$ & 0.098 & Amp.\&Pot. & Yes \\
\hline
\end{tabular}

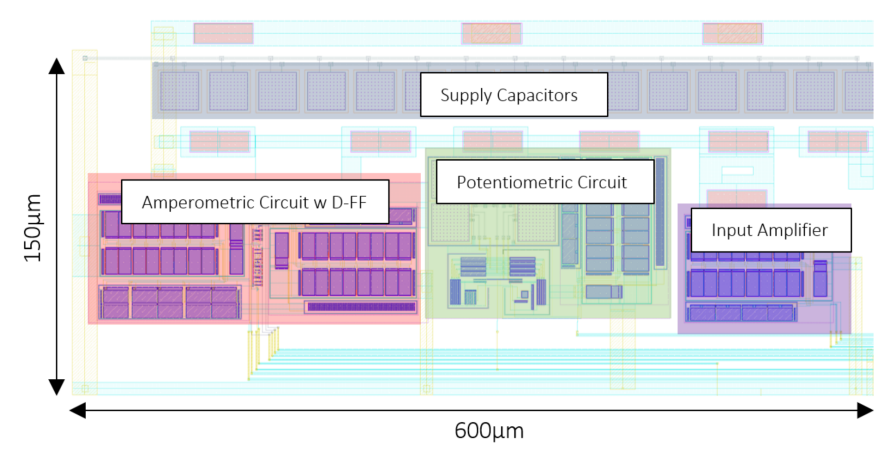

Fig. 7: Layout of DAPPER

\section{Simulation Results}

The circuit is designed in a standard 180nm CMOS technology and simulated with cadence. The layout is presented in Fig. 7 with the circuits occupies a total area of $0.098 \mathrm{~mm}^{2}$. Fig. 6 displays the output frequency ranges for both the potentiometric and the amperometric circuits. For the amperometric circuit, the frequency output is shown for DC current inputs from $250 \mathrm{pA}$ to $5.6 \mu \mathrm{A}$. This circuit draws $29 \mu \mathrm{W}$ at the maximum frequency. The output frequency of the D flip-flop when potentiometry is deactivated begins at $214 \mathrm{~Hz} @ 250 \mathrm{pA}$ before increasing to $551 \mathrm{kHz} @ 5.6 \mu \mathrm{A}$.

The frequency output shows a linear trend from 250pA to $0.1 \mathrm{uA}$ with a slope of $1260 \mathrm{~Hz} / \mathrm{nA}$. From $0.1 \mathrm{uA}$ to $5.6 \mu \mathrm{A}$, this gradient reduces to $68 \mathrm{~Hz} / \mathrm{nA}$. This corresponds with the reduced gain of the amplifier after the bandwidth of $10 \mathrm{kHz}$ of the amplifier. The propagation delay $t_{p d}$ from the comparator output to $\mathrm{S} 1$ increases from $100 \mathrm{~ns}$ at $I_{i n}=250 \mathrm{pA}$ to $500 \mathrm{~ns}$ at $I_{i n}=0.1 \mu \mathrm{A}$, which explains the difference in the gradient as per Eq. 3. This delay increases as for higher currents, $C_{I N T}$ takes a longer time to discharge.

The simulated input-output characteristics of the potentiometric circuit is shown in Fig. 6, showing sufficiently linear behavior between $0.4 \mathrm{~V}$ to $1 \mathrm{~V}$, which is adequate for values measured using a metal oxide based $\mathrm{pH}$ electrode [15]. The curve displays a linear relation, with a slope of $-11.4 \mathrm{~Hz} / \mathrm{V}$ input. The power consumption at the maximum frequency of the circuit is $3 \mu \mathrm{W}$.

Fig. 8 displays the output simulation for the D flip-flop

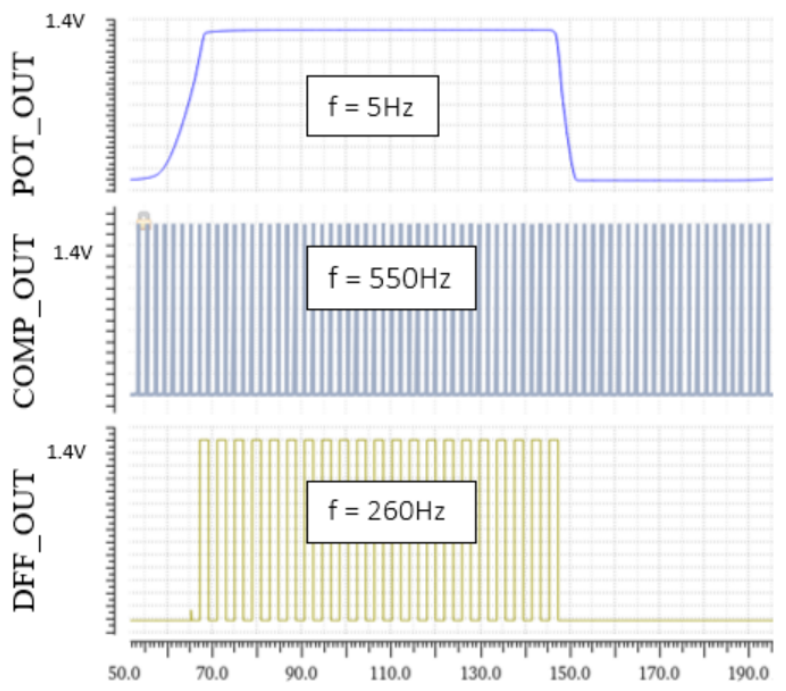

Fig. 8: Simulation of the output voltages for an Isense $=300 \mathrm{pA}$ and Vsense $=0.7 \mathrm{~V}$. This corresponds to $5 \mathrm{~Hz}$ and $260 \mathrm{~Hz}$ on the D flip-flop output.

for Isense $=300 \mathrm{pA}$ and Vsense $=0.7 \mathrm{~V}$. The reset switch is effective in modulating the output of the D flip-flop to correspond to the potentiometric output frequency, while the amperometric output frequency can still be obtained from the individual pulses.

A comparison with state-of-the-art is presented in Table. I demonstrating the innovative aspect of this work in providing concurrent dual transduction methodologies. The chip is now in fabrication. The future plan is to test the performance of the chip with both off-chip and on-chip glucose and $\mathrm{pH}$ sensors.

\section{CONClusion And Future Work}

An analog front-end chip capable of simultaneous readings of potentiometry and amperometry is presented along with the design of each system block. The output consists of two frequencies that vary according to the input current and voltage that can be transmitted wirelessly through backscattering. Simulation results are presented to demonstrate the performance and functionality of the chip. 


\section{REFERENCES}

[1] A. Ainla, M. P. Mousavi, M.-N. Tsaloglou, J. Redston, J. G. Bell, M. T. Fernandez-Abedul, and G. M. Whitesides, "Open-source potentiostat for wireless electrochemical detection with smartphones," Analytical chemistry, vol. 90, no. 10, pp. 6240-6246, 2018.

[2] N. Moser, T. S. Lande, C. Toumazou, and P. Georgiou, "Isfets in cmos and emergent trends in instrumentation: A review," IEEE Sensors Journal, vol. 16, no. 17, pp. 6496-6514, 2016.

[3] M. Diabetes, "Medtronic enlite glucose sensors," 10 January 2020. [Online]. Available: https://www.medtronic-diabetes.co.uk/continuousglucose-monitoring/enlite-sensor

[4] S. Baliga, S. Muglikar, R. Kale et al., "Salivary ph: A diagnostic biomarker," Journal of Indian Society of Periodontology, vol. 17, no. 4, p. 461, 2013.

[5] S. Kumar, S. Padmashree, and R. Jayalekshmi, "Correlation of salivary glucose, blood glucose and oral candidal carriage in the saliva of type 2 diabetics: A case-control study," Contemporary clinical dentistry, vol. 5, no. 3, p. 312, 2014.

[6] A. J. Bard and L. R. Faulkner, Electrochemical methods : fundamentals and applications / Allen J. Bard, Larry R. Faulkner. Wiley New York, 1980.

[7] J. E. Choi, J. N. Waddell, K. Lyons, and J. A. Kieser, "Intraoral ph and temperature during sleep with and without mouth breathing." Journal of oral rehabilitation, vol. 43 5, pp. 356-63, 2016.

[8] M. J. Schöning, R. Krause, K. Block, M. Musahmeh, A. Mulchandani, and J. Wang, "A dual amperometric/potentiometric fia-based biosensor for the distinctive detection of organophosphorus pesticides," Sensors and Actuators B: Chemical, vol. 95, no. 1-3, pp. 291-296, 2003.

[9] A. Sun, A. Venkatesh, and D. A. Hall, "A multi-technique reconfigurable electrochemical biosensor: Enabling personal health monitoring in mobile devices," IEEE transactions on biomedical circuits and systems, vol. 10, no. 5, pp. 945-954, 2016.

[10] H. Jiang, X. Zhou, S. Kulkarni, M. Uranian, R. Seenivasan, and D. A. Hall, "A sub-1 $\mu$ w multiparameter injectable biomote for continuous alcohol monitoring," in 2018 IEEE Custom Integrated Circuits Conference (CICC). IEEE, 2018, pp. 1-4.

[11] A. Cavallini, C. Baj-Rossi, S. Ghoreishizadeh, G. De Micheli, and S. Carrara, "Design, fabrication, and test of a sensor array for perspective biosensing in chronic pathologies," in 2012 IEEE Biomedical Circuits and Systems Conference (BioCAS). IEEE, 2012, pp. 124-127.

[12] P. Feng, P. Yeon, Y. Cheng, M. Ghovanloo, and T. G. Constandinou, "Chip-scale coils for millimeter-sized bio-implants," IEEE transactions on biomedical circuits and systems, no. 99, pp. 1-12, 2018.

[13] S. S. Ghoreishizadeh, I. Taurino, G. De Micheli, S. Carrara, and P. Georgiou, "A differential electrochemical readout asic with heterogeneous integration of bio-nano sensors for amperometric sensing," IEEE transactions on biomedical circuits and systems, vol. 11, no. 5, pp. 11481159, 2017.

[14] W. O. Ting and S. S. Ghoreishizadeh, "Autonomous readout asic with $169 \mathrm{db}$ input dynamic range for amperometric measurement," in 2018 25th IEEE International Conference on Electronics, Circuits and Systems (ICECS). IEEE, 2018, pp. 869-872.

[15] D. Ma, C. Mason, and S. Ghoreishizadeh, "A wireless system for continuous in-mouth ph monitoring," in 2017 IEEE Biomedical Circuits and Systems Conference, BioCAS 2017-Proceedings. IEEE, 2018.

[16] S. Dai, R. T. Perera, Z. Yang, and J. K. Rosenstein, "A 155-db dynamic range current measurement front end for electrochemical biosensing," IEEE transactions on biomedical circuits and systems, vol. 10, no. 5, pp. 935-944, 2016.

[17] L. B. Leene, M. Maslik, P. Feng, K. M. Szostak, F. Mazza, and T. G. Constandinou, "Autonomous soc for neural local field potential recording in mm-scale wireless implants," in 2018 IEEE International Symposium on Circuits and Systems (ISCAS). IEEE, 2018, pp. 1-5.

[18] A. Devices, "Ad5940-41 datasheet," 10 January 2020. [Online]. Available: https://www.analog.com/en/products/ad5941.html?doc=AD59405941.pdf 